\title{
Las políticas de salud vistas desde el género: Una aproximación a partir del examen de los planes de control del tabaquismo
}

\author{
María Luisa JIMÉNEZ RODRIGO \\ Departamento de Sociología \\ Universidad de Sevilla \\ mljimenez@us.es
}

Recibido: 24.07 .2014

Aceptado: 09.01.2015

\section{RESUMEN:}

Aunque es numerosa la literatura que advierte sobre la presencia de sesgos de género en las políticas de salud, las políticas de prevención y atención al consumo de tabaco, pese al proceso de feminización de esta práctica, han sido objeto de escasa reflexión en España desde una mirada crítica de género. Este trabajo centra su interés en el análisis de los sesgos de género e interseccionales que pueden afectar al diseño de los planes de control del tabaquismo desarrollados a nivel regional y nacional en España. Si bien la mayoría de los planes incorpora datos sobre la situación desagregados por sexo, las mujeres permanecen invisibles en la determinación de objetivos y medidas de intervención más allá del embarazo. Igualmente, no se contemplan necesidades de perfiles específicos en relación al género y otros ejes de desigualdad.

Palabras clave: Evaluación de políticas; Sesgos de género; Interseccionalidad; Desigualdades en salud; Consumo de tabaco.

\section{Health policies from a gender perspective: An approach from the exam of tobacco control plans}

\begin{abstract}
Although research on gender biases in health policies is extensive, tobacco control policies, spite of smoking feminization processes, have been scarcely examined in Spain from a critical gender lens. This paper focuses on the analysis of gender and intersectional biases that could affect the design of tobacco control plans as have been developed at national and regional levels. Even though sexsegregated data is mandatorily incorporated in the majority of plans, women specific characteristics remain invisible in the setting of objectives and interventions with the exception of pregnancy. Consequently, there is not drawing of distinct profiles that take into account gender intersection with other inequality axes.
\end{abstract}

Keyword: Policy Evaluation; Gender Biases; Interseccionality; Health Inequalities; Smoking. 


\section{INTRODUCCIÓN}

La incorporación de la perspectiva de género en las políticas públicas constituye un paso esencial e ineludible para el avance efectivo en la igualdad entre mujeres y hombres. Por ello, desde diferentes agencias políticas, académicas y sociales se insta a la transversalización del género en las políticas, de forma que éstas puedan funcionar con efecto transformador en las relaciones de género, esto es, que sean "sensitivas" o "inclusivas" al género (García Prince, 2008: 60). La invisibilidad de las necesidades y problemas de las mujeres, su reducción a roles reproductivos y familiares, la definición de los problemas públicos en términos fundamentalmente masculinos son prácticas que suelen afectar a las políticas "ciegas al género", provocando que éstas tengan efectos no equivalentes en mujeres y en hombres, y reproduciendo e, incluso, reforzando las estructuras existentes de poder basadas en el género (Lombardo, Meier y Verloo, 2012).

Son numerosos los trabajos que advierten de la presencia de sesgos de género en las políticas de salud y de sus consecuencias en la invisibilización de las situaciones de salud de las mujeres así como en el entendimiento y atención parcial de sus problemas y necesidades (Peiró et al., 2004; Sen, Östlin y George, 2005; Valls, 2009, GarcíaCalvente, Jiménez Rodrigo y Martínez Morante, 2010). Sin embargo, las políticas de prevención y atención al consumo de tabaco, pese al proceso de feminización de esta práctica y su incremento en determinados perfiles de mujeres, han sido objeto de escasa reflexión en España desde una mirada crítica de género. $Y$ es paradójico, pues el consumo de tabaco constituye un importante factor de desigualdad social y de género en salud, determinándose el consumo de tabaco entre mujeres y chicas jóvenes como un problema prioritario de salud pública (WHO, 1999, 2007).

En España, el consumo de tabaco ha experimentado desde los años setenta del pasado siglo un significativo proceso de feminización, extendiéndose progresivamente entre mujeres de distintos niveles socioeconómicos y grupos de edad (Jiménez Rodrigo, 2010, 2012). Actualmente, fuman de forma habitual un $20,2 \%$ de las mujeres mayores de 16 años frente al $27,9 \%$ de los varones. Mientras que el consumo masculino de tabaco se ha reducido desde 1995 en más de 15 puntos porcentuales, el de las mujeres apenas ha descendido en ese periodo en poco más de cuatro puntos. Pese a esta mínima reducción global del consumo de tabaco entre las mujeres, determinados perfiles están experimentando un incremento y una concentración del consumo de tabaco. Es el caso de las mujeres mayores de 45 años, cuyo consumo de cigarrillos ha crecido desde 2003 a $2011-12$ del 25,5\% al 
$30 \%$ en el grupo de $45-54$ años, y del 7,4\% al 15,4\% en el grupo de $55-64$ años ${ }^{1}$. Se está produciendo, por tanto, un envejecimiento del perfil de las mujeres fumadoras, ya que éstas no están logrando dejar de fumar como sí se está observando en sus coetáneos varones. Por otro lado, las chicas entre 14 y 18 años, continúan fumando más que los chicos, con una prevalencia de consumo en el último mes de un 29,3\% frente al 23\% (PNSD, 2010). Estos datos se corresponden con un incremento de la morbimortalidad femenina relacionada con el consumo de tabaco (Sánchez de Cos, 2009).

Las tendencias de consumo de tabaco, además de estar marcadas por el género y la edad, están estructuradas por la clase social, la raza/etnia y el origen nacional. Entre los hombres, el consumo de tabaco se concentra en las clases trabajadoras manuales y varones en desempleo, registrándose una significativa reducción entre las clases sociales medias-altas y varones con estudios universitarios. Entre las mujeres, se observa un aumento del consumo de tabaco entre las clases trabajadoras no cualificadas, mujeres en desempleo y con estudios medios así como una disminución entre las ocupaciones directivas. Por otra parte, las mujeres de origen extranjero muestran una prevalencia del 18,3\% frente al $26,5 \%$ de los varones ${ }^{2}$. Entre la población gitana, la brecha de género es ampliamente significativa, pues el consumo de tabaco es considerablemente menor entre las mujeres gitanas, con una tasa de $14,7 \%$ frente al $54,9 \%$ de los varones gitanos (Laparra et al., 2011).

Las transformaciones en las pautas del consumo de tabaco así como la complejidad social que marca la intersección del género con otros ejes de desigualdad implican decisivos retos a las políticas en salud en el logro de la equidad. Sin embargo, y a la vista de los datos, no son pocas las críticas que han venido cuestionando la eficacia y la equidad de género de las políticas de prevención y atención al consumo de tabaco y sus desiguales efectos en mujeres y hombres (Oakley, 1989; Greaves y Barr, 1999, Christofides, 2001; Dedobbeleer et al., 2004; Greaves y Jategaonkar 2006; Amos et al., 2012). Es en esta discusión donde emerge el interés de este trabajo en el análisis de los sesgos de género que pueden afectar a las políticas sobre tabaco en España, concretamente en relación a su materialización en los planes integrales de tabaquismo ${ }^{3}$. La revisión de estos

${ }^{1}$ Instituto Nacional de Estadística y Consulta Interactiva del Sistema Nacional de Salud a partir de los datos de las Encuestas Nacionales de Salud (1993, 2003, 2011/12).

${ }^{2}$ Instituto Nacional de Estadística a partir de los datos de las Encuestas Nacionales de Salud (2003, 2011/12).

3 Distinguimos entre los términos consumo de tabaco y tabaquismo. El primero hace referencia a la práctica social y el segundo a su acepción biomédica más restringida que alude a su definición como enfermedad crónica perteneciente al grupo de las adicciones y 
planes nos servirá como (pre)texto para poner sobre la mesa la pertinencia de la incorporación de un enfoque de género en las políticas de salud, no sólo para avanzar en equidad sino también en eficiencia, cuestiones especialmente sensibles en un contexto de crisis económica y de reducción de las inversiones públicas en salud.

Este trabajo presenta, en primer lugar, un repaso de la literatura que ha abordado el estudio de los principales sesgos de género que afectan a las políticas en salud para concretar en cómo éstos se presentan en las políticas de control del consumo de tabaco. A continuación, se describe el proceso metodológico seguido a partir de una estrategia de análisis documental de evaluación del grado de sensibilidad de género en diferentes aspectos del diseño de los planes de control del tabaquismo. Los resultados aquí expuestos revelan que, pese a la generalizada incorporación del sexo como variable en los diagnósticos de la situación, la salud de las mujeres continúa reduciéndose a lo sexual-reproductivo, focalizándose el interés de las intervenciones en el problema del "tabaquismo materno".

\section{SESGOS DE GÉNERO EN LA POLÍTICAS DE SALUD}

La presencia de sesgos de género en la investigación en salud ha sido ampliamente señalada y discutida (Ruiz Cantero y Verbrugge, 1997; Doyal, 2000; Inhorn y Whitlle, 2001; Valls, 2009; Nieuwenhoven y Klinge, 2010) así como su reproducción en las políticas e intervenciones sanitarias (Peiró et al., 2004; Sen, Östlin y George, 2005; Valls, 2009, García Calvente, Jiménez Rodrigo y Martínez Morante, 2010). Los principales problemas de género derivan principalmente de dos prácticas: una primera, cuando se asumen situaciones y riesgos de salud que son diferentes para mujeres y hombres como similares; y la segunda, cuando se entienden como radicalmente diferentes situaciones similares en salud para mujeres y para hombres (Ruiz Cantero y Verbrugge, 1997).

De la primera práctica emerge el androcentrismo, que implica la adopción de una perspectiva masculina -especialmente, la de los varones occidentales, blancos y de clase media- en la interpretación de la realidad (Ortiz, 2006: 42), lo que conlleva el entendimiento parcial y sesgado de los problemas de salud entre las mujeres así como su invisibilización en enfermedades, dolencias y problemas de salud tradicionalmente vistos como masculinos. Esto se ha evidenciado, en el caso de enfermedades cardiovasculares y en determinados tipos de cáncer-como el de pulmón o colorrectal-, en la exclusión sistemática de las mujeres en los ensayos

catalogada en el Manual Diagnóstico y Estadístico de los Trastornos Mentales DSM-IV de la Asociación Americana de Psiquiatría. 
clínicos y en la persistencia de sesgos diagnósticos y terapéuticos en la atención de las necesidades en salud de las mujeres (Ruiz Cantero y Verdú, 2004; Valls, 2009).

La configuración del tabaquismo como problema de salud pública no ha escapado del androcentrismo. El grueso de la investigación se ha dirigido hacia dos enfermedades que tradicionalmente han sido consideradas como masculinas: el cáncer de pulmón y las enfermedades cardiovasculares ${ }^{4}$, donde todavía persiste una infrarrepresentación de las mujeres en las muestras de los estudios clínicos (Jagsi et al., 2009). Así también, se ha analizado cómo las campañas de sensibilización de los riesgos del consumo de tabaco fueron delineadas y enfocadas principalmente en función de las necesidades de los varones (Waldron, 1991; Greaves y Barr, 1999). Este androcentrismo también se manifiesta en la atención sanitaria, por ejemplo, en la desigual distribución del consejo médico para dejar de fumar entre mujeres y hombres (Jiménez Rodrigo, 2011).

En interacción con el androcentrismo, se ha criticado el problema de la insensibilidad de género que continúa afectando a las políticas e investigaciones en salud (Bird y Rieker, 1999; Doyal, 2000; Peiró et al., 2004; Sen, Östlin y George, 2005) y, también, a las políticas sobre tabaquismo (Greaves y Barr, 1999; Christofides, 2001; Dedobbeleer et al., 2004; Amos et al., 2012). Estos aspectos podrían explicar el menor impacto de los mensajes sanitarios entre las mujeres sobre todo, entre aquéllas con escasos recursos y entre las más jóvenes- (Jacobs, 2001; Greaves y Jategaonkar 2006) y las desiguales tendencias de abandono observadas en mujeres y en hombres (Jacobs, 2001).

La segunda práctica nociva a la sensibilidad de género que consiste en la asunción de diferencias en salud entre mujeres y hombres se sustenta en el reduccionismo biológico de la salud femenina y su focalización a su dimensión sexual-reproductiva (Ruiz Cantero y Verbrugge, 1997; Im, 2000). La esencialización biológica de los problemas de salud de las mujeres ofrece una visión simple y descontextualizada de éstas como sexo reproductor, quedando su salud determinada por el embarazo, el parto y otros aspectos de la maternidad (Inhorn y Whittle, 2001). Son esferas que, además, se encuentran fuertemente

\footnotetext{
${ }^{4}$ Esta relación se construye desde los primeros ensayos clínicos tras la Segunda Guerra Mundial que constataron la causalidad entre consumo crónico de tabaco y cáncer de pulmón. Pero esta relación sólo se confirmó en la población masculina ya que las muestras estaban compuestas exclusivamente por varones (Hirschfelder, 1999). Esta práctica se justificaba fundamentalmente porque "las relativamente pocas mujeres fumadoras, en general, no han fumado tanto tiempo ni con tanta intensidad como los varones fumadores, así que ellas no proporcionan información directa sobre los efectos del consumo de tabaco a largo plazo" (Doll et al., 1994: 901).
} 
medicalizadas y controladas sanitariamente (Barker, 1998), tornando procesos naturales como el embarazo o el parto en procesos patológicos que requieren de la supervisión médica para su normalización social (Cahill, 2001). Igualmente, tanto las políticas como la investigación sobre mujeres y consumo de tabaco se han centrado en su faceta sexual-reproductiva. La restricción de los efectos del consumo de tabaco entre las mujeres durante el embarazo y sobre el feto ha sido el pilar fundamental sobre el cual se ha construido el problema del "tabaquismo femenino" como problema de salud pública (Oakley, 1989; Berridge, 2001; Christofides, 2001).

La esencialización biológica de la salud de las mujeres implica no solo la ignorancia de la dimensión de género sino también la omisión de otros procesos causales interactivos entre el género con otros estratificadores sociales como la edad, la clase social, la etnicidad o la orientación sexual (Sen, Östlin y George, 2005). En este sentido, cobran fuerza nuevos enfoques sobre las desigualdades en salud basados en el marco interseccional (Bowleg, 2012; Hankivsky, 2012; Bauer, 2014). Este enfoque propone el análisis de las categorías sociales múltiples a partir de las interrelaciones entre género, etnia/raza, clase social, identidad sexual (principalmente, y entre otras) para examinar cómo éstas intersectan en las experiencias individuales y reflejan el solapamiento de múltiples sistemas de privilegio y opresión (sexismo, racismo, clasismo y heterosexismo, entre otros) (Bowleg, 2012; Guzmán, 2014). Este enfoque promueve una mayor atención a la heterogeneidad de efectos y procesos causales que producen desigualdades en salud (Hankivsky, 2012). Desde una mirada interseccional, los principales sesgos identificados están fundamentalmente relacionados con la invisibilidad interseccional de posiciones marginalizadas en la configuración de las problemáticas en salud al ser excluidas de las políticas e intervenciones, como se ha visto, por ejemplo, en relación al uso de drogas (Jiménez Rodrigo y Guzmán, 2012) o al VIH/SIDA (Brëdstrom, 2006; Doyal, 2009).

\section{APUNTES METODOLÓGICOS}

Los textos institucionales como productos sociales reflejan y son reflejo de las relaciones sociales en las que emergen. Por ello, los tomamos como topos de representación de los sesgos de género que pueden afectar a las políticas públicas. Estos textos, políticamente mediados, muestran, a su vez, el proceso de construcción social de los problemas sociales y públicos y cómo son representadas sus causas y consecuencias, qué actores intervienen y qué sujetos y qué objetos de interevención son señalados a partir de la definición de las políticas públicas. De tal forma, el examen de los textos institucionales permite indagar en los marcos interpretativos de las políticas sobre cómo se configura el problema y las soluciones que adoptan las políticas (Bustelo y Lombardo, 2007). 
Desde esta perspectiva constructivista de los problemas sociales y de las políticas públicas, enmarcamos el análisis que aquí presentamos de los planes de tabaquismo a partir de un análisis de los sesgos de género. Para ello, partimos de la propuesta analítica de Magrit Eichler y Mary Anne Burke (Eichler, 1999; Eichler y Burke, 2006) para la identificación y eliminación de sesgos de género en el ámbito de la investigación en salud y que, en este trabajo, ha sido adaptada para el análisis de los textos de las políticas de salud. Estos sesgos derivan de tres grandes problemas: el androcentrismo, la insensibilidad a las diferencias entre los sexos y los dobles estándares.

En primer lugar, el androcentrismo conlleva la adopción de una perspectiva masculina a la hora de entender los problemas sociales, favoreciendo la normalización de las experiencias masculinas y la invisibilización o patologización de las femeninas. En el ámbito de las políticas públicas, el androcentrismo puede resguardarse bajo una pretendida "neutralidad de género" que tiende a reproducir las normas y las situaciones masculinas a la hora de entender las problemáticas sociales, ignorando o poniendo en desventaja las femeninas (Lombardo, Meier y Verloo, 2012: 12).

En segundo lugar, la insensibilidad a las diferencias entre los sexos consiste en ignorar el sexo y el género en contextos en donde son significativos, produciéndose la infrarrepresentación o exclusión de algún sexo. Una manifestación habitual de este sesgo es la descontextualización de las experiencias femeninas y masculinas al no atender al marco social, económico y cultural en el que se desenvuelven las relaciones de género. Otras prácticas relacionadas con este problema son la sobregeneralización o universalización y la asunción de la homogeneidad dentro de grupos.

En tercer lugar, los dobles estándares implican tratar y evaluar situaciones sustancialmente parecidas o idénticas como diferentes en función del sexo. Los dobles estándares pueden manifestarse en el dicotomismo sexual al entender a los dos sexos como opuestos, exagerando las diferencias entre mujeres y hombres, sin que sus características se solapen o influyan el uno en el otro. Esto puede materializarse tanto en la hiperrepresentación de un sexo como en la reificación de estereotipos de género, convirtiéndolos en características naturales e innatas a los sexos.

Si bien el análisis de género nos puede ayudar a revelar situaciones que invisibilizan y/o deforman las situaciones de las mujeres -y de los hombres-, también puede eclipsar experiencias y posiciones sociales múltiples ubicadas en la intersección del género con otros ejes de desigualdad (Weldon, 2006). Por ello, incorporamos a nuestro esquema analítico las aportaciones del marco interseccional 
en el campo de las políticas públicas, que se pregunta cómo éstas consideran a los grupos en sus intersecciones de desigualdad (Crenshaw, 1991). A este respecto, nos planteamos si las políticas sobre tabaquismo entienden a mujeres y a hombres como un todo o contemplan diferentes necesidades y problemas en función de categorías sociales múltiples ${ }^{5}$. Atenderemos, además, a cómo se visibilizan, explicitan e incluyen determinadas desigualdades sociales en las políticas, al mismo tiempo que pueden obviarse otras [invisibilidad interseccional] (Jiménez Rodrigo y Guzmán, 2012: 88). El señalamiento en las políticas de determinadas categorías interseccionalizadas también puede contribuir a la estigmatización y reforzamiento de estereotipos [estereotipificación múltiple] sobre determinados grupos así como a la representación privilegiada de grupos dominantes y de sus intereses (Roggeband y Verloo, 2007; Lombardo y Rolandsen Agustín, 2012).

A partir de este esquema, se diseñó una herramienta de análisis de sesgos de género e interseccionales (cuadro 1) que puede aplicarse a diferentes componentes del diseño de los planes de implementación de las políticas: definición del problema, diagnóstico de la situación, objetivos y metas, medidas e intervenciones y evaluación (Subirats et al., 2008). En primer lugar, el análisis de la definición del problema público implica examinar el proceso de construcción de los argumentos a través de los cuales se convierte una condición en problema y cómo éste es representado, atribuyendo causas, culpas y responsabilidades. Esta "historia causal" contiene los argumentos de persuasión a otros actores y a la opinión pública de que este problema debe ser objeto de intervención pública (Stone, 1989). En segundo lugar, atenderemos a qué datos se emplean dentro de los diagnósticos para justificar los objetivos y medidas de intervención propuestas para solucionar el problema. Dependiendo de qué datos se empleen para la definición de la realidad se mostrará -o no- la existencia de diferencias entre mujeres y hombres, pudiendo interpretarse -o no- en términos de desigualdad (Astelarra, 2004). En tercer lugar, se tendrá en cuenta cómo los planes identifican y caracterizan los grupos objeto de intervención. Éste es un aspecto fundamental en el análisis de las "historias causales" que sostienen las políticas públicas, pues la definición de poblaciones diana supone la atribución de determinadas características, valores, imágenes y símbolos que distinguen a estos grupos como socialmente significativos o vulnerables, ubicándolos en una posición privilegiada o dependiente (Schneider e Ingram, 1993). Junto a estos elementos de diagnosis, otros componentes fundamentales del diseño de las políticas son los referidos a la prognosis o pronóstico, es decir, la definición sobre cómo ha de tratarse el problema (Bustelo y Lombardo, 2007). Aquí se

${ }^{5}$ Ange-Mary Hanckok (2007: 64) distingue entre categorías "múltiples"-cuando se emplea más de una categoría, pero de forma estática y uniforme- e "interseccionales" - cuando las categorías mantienen una relación abierta, dinámica y diversa-. 
atenderá a cómo son definidos los objetivos y metas de la política así como son determinadas las diferentes medidas, acciones e instrumentos de intervención planeados para su consecución. Por último, se analizarán los procedimientos de evaluación e indicadores que contemplan los diferentes planes para la medición de su efectividad.

Cuadro 1. Matriz de análisis de sesgos de género e interseccionales en las políticas de salud

\begin{tabular}{|c|c|c|}
\hline Tipos de sesgos & Cuestiones de análisis & $\begin{array}{l}\text { Componentes de las } \\
\text { políticas }\end{array}$ \\
\hline $\begin{array}{c}\text { ANDROCENTRIS } \\
\text { MO }\end{array}$ & $\begin{array}{l}\text { Normalización: ¿Lo masculino se define como la } \\
\text { norma en función de la cual se evalúan las } \\
\text { experiencias femeninas? } \\
\text { Patologización: ¿Se patologiza las experiencias de } \\
\text { las mujeres? }\end{array}$ & \\
\hline $\begin{array}{l}\text { INSENSIBILIDAD } \\
\text { A LAS } \\
\text { DIFERENCIAS } \\
\text { ENTRE LOS } \\
\text { SEXOS }\end{array}$ & $\begin{array}{l}\text { Insensibilidad a la diferencia: ¿Se ignoran las } \\
\text { diferencias entre mujeres y hombres? } \\
\text { Infrarrepresentación / exclusión: ¿Algún sexo está } \\
\text { infrarrepresentado o excluido? } \\
\text { Descontextualización ¿Se omite el contexto social, } \\
\text { económico, político y cultural? } \\
\text { Sobregeneralización / Universalización: ¿La } \\
\text { información sobre un sexo se aplica } \\
\text { automáticamente al otro? }\end{array}$ & $\begin{array}{l}\text { - Definición del } \\
\text { problema } \\
\text { - Diagnóstico } \\
\text { - Identificación y } \\
\text { caracterización } \\
\text { de los grupos }\end{array}$ \\
\hline $\begin{array}{l}\text { DOBLES } \\
\text { ESTÁNDARES }\end{array}$ & 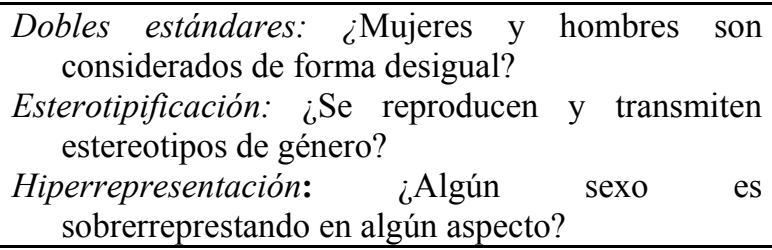 & 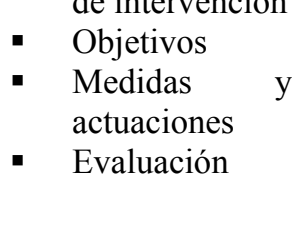 \\
\hline $\begin{array}{c}\text { SESGOS } \\
\text { INTERSECCIO } \\
\text { NALES }\end{array}$ & $\begin{array}{l}\text { Invisibilidad interseccional: ¿Se consideran las } \\
\text { categorías interseccionalizadas? ¿Se } \\
\text { hipervisibilizan/invisibilizan ciertas categorías? } \\
\text { Estereotipificación múltiple: ¿Se transmiten } \\
\text { estereotipos vinculados a categorías múltiples? }\end{array}$ & \\
\hline
\end{tabular}

Fuente: Elaboración propia

El corpus documental está conformado todos por los planes nacionales y regionales específicos (integrales o sectoriales) sobre tabaquismo publicados en España y disponibles en las páginas web institucionales ${ }^{6}$ a fecha de 1 de mayo de 2014 (véase cuadro 2).

${ }^{6}$ Se excluyen en la delimitación del corpus documental los planes genéricos sobre salud y drogodependencias en donde algunas Comunidades Autónomas incluyen cuestiones 
Cuadro 2. Planes sobre control de tabaquismo incluidos en el análisis

\begin{tabular}{|c|c|c|c|}
\hline Nacional & $\begin{array}{l}\text { Plan Nacional de Prevención y } \\
\text { Control del Tabaquismo }\end{array}$ & $2003-2007$ & NAC-03/07 \\
\hline \multirow{2}{*}{ Andalucía } & $\begin{array}{l}\text { Plan de Actuación sobre el } \\
\text { Tabaquismo en Andalucía }\end{array}$ & 2002 & AND-02 \\
\hline & $\begin{array}{c}\text { Plan Integral de Tabaquismo de } \\
\text { Andalucía }\end{array}$ & $2005-2010$ & AND-05/10 \\
\hline Asturias & $\begin{array}{l}\text { Programa de Control y Prevención } \\
\text { del Tabaquismo }\end{array}$ & 2009-2012 & AST-09/12 \\
\hline Baleares & $\begin{array}{l}\text { Pla sobre el Tabaquisme a Les Illes } \\
\text { Balears }\end{array}$ & $2003-2007$ & BAL-03/07 \\
\hline \multirow{3}{*}{ Cantabria } & $\begin{array}{l}\text { I Plan de Prevención y Control del } \\
\text { Tabaquismo en Cantabria }\end{array}$ & 2004-2007 & CAN-04/07 \\
\hline & $\begin{array}{l}\text { II Plan de Prevención y Control del } \\
\text { Tabaquismo }\end{array}$ & $2008-2011$ & CAN-08/11 \\
\hline & $\begin{array}{l}\text { III Plan de Prevención y Control del } \\
\text { Tabaquismo }\end{array}$ & $2012-2016$ & CAN-12/16 \\
\hline $\begin{array}{r}\text { Castilla-La } \\
\text { Mancha }\end{array}$ & $\begin{array}{l}\text { Plan de Prevención y Tratamiento } \\
\text { Del } \\
\text { Tabaquismo en Castilla La Mancha }\end{array}$ & $2002-2007$ & CLM-02/07 \\
\hline Ceuta & III Plan Integral de Tabaquismo & 2013-2017 & CEU-13/17 \\
\hline Madrid & $\begin{array}{l}\text { Plan Regional de Prevención y } \\
\text { Control del Tabaquismo en la } \\
\text { Comunidad de Madrid }\end{array}$ & $2005-2007$ & MAD-05/07 \\
\hline Rioja & Plan Riojano de Tabaquismo & $2009-2013$ & $\mathrm{RIO}-09 / 13$ \\
\hline
\end{tabular}

Fuente: Elaboración propia

Los planes fueron analizados tanto cualitativa como cuantitativamente. El análisis cualitativo se llevó a cabo mediante un proceso de indagación y codificación de los textos de los planes siguiendo las categorías y cuestiones recogidas en la matriz de análisis (cuadro 1). Para ello, se empleó el programa Atlas.ti 7, que facilita la codificación de los documentos y la recuperación de las citas significativas para su posterior interpretación. Desde el punto de vista

relativas al control del tabaquismo, como es el caso de Aragón, Castilla León, Galicia o País Vasco (planes de drogodependencias) o el de Valencia (plan de salud). 
cuantitativo, se diseñaron unas listas de verificación para registrar el grado de sensibilidad al género de diferentes componentes del diseño de los planes. Respecto al diagnóstico de la situación, se examinó el uso de las categorías ligadas al sexo -si éstas se presentaban como categorías únicas o de forma múltiple en interrelación con otras, como la edad, la clase social o la nacionalidad- y cómo éstas se aplicaban al estudio de las variables escogidas para el diagnóstico. Por otra parte, se evaluó el grado de sensibilidad y especificidad de género en la redacción de los objetivos así como en el planteamiento de las medidas y procedimientos de evaluación. Por objetivos, medidas e indicadores sensibles al género entendemos aquéllos que incorporan en sus planteamientos la necesidad de abordar las diferencias y desigualdades entre mujeres y hombres en relación a las necesidades en salud. Los objetivos, medidas e indicadores específicos al género tratarían de clarificar necesidades particulares de salud y de atención de mujeres o de varones (García Calvente, Jiménez Rodrigo y Martínez Morante, 2010: 73).

\section{RESULTADOS}

\subsection{El tabaquismo como problema: De la invisibilidad de las mujeres a la construcción del tabaquismo materno.}

El tabaquismo como problema de salud pública representado en los planes emana de la definición de las instituciones sanitarias internacionales de referencia (en particular, la Organización Mundial de la Salud) mediante de tres argumentaciones fundamentales. Primero, a partir de la vinculación entre consumo de tabaco y morbimortalidad (principalmente, cáncer de pulmón y enfermedades cardiovasculares), definiéndose el tabaquismo como primera causa prevenible de mortalidad en los países "desarrollados". Pese a que las estadísticas muestran una importante prevalencia de consumo de tabaco entre las mujeres, son escasos exceptuando alguna alusión a las mujeres en relación a las estrategias de la industria tabaquera y a los condicionantes sociales que pueden afectar al uso femenino de tabaco- los planes que incorporan en la configuración del problema las situaciones de las mujeres fumadoras. Esto ocasiona no sólo una invisibilización de éstas sino también la reproducción de cierto androcentrismo implícito, que continúa identificando los riesgos del consumo de tabaco con enfermedades social y médicamente masculinizadas. El empleo del masculino como genérico contribuye, además, al sostenimiento de esta asociación.

Segundo, el problema del tabaquismo hace referencia a la adicción al tabaco provocada principalmente por la nicotina, que acaba condicionando su abuso. Se entiende, entonces, el tabaquismo como una enfermedad crónica ligada a la adicción. La reducción del consumo del tabaco a sus aspectos adictivos conlleva la omisión de otros factores sociales y de género que condicionan las carreras de 
consumo y de abandono (Waldron, 1991; Dedobbeleer et al., 2004; Greaves y Jategaonkar, 2006; Jiménez Rodrigo, 2011).

Tercero, junto a las pérdidas en salud, el problema del tabaquismo es definido en función de los costes económicos que éste conlleva en gastos sanitarios y años de vida "productiva" perdidos así como en relación a problemas derivados como el contrabando. Igualmente, en tales argumentaciones no se hace referencia ni a la influencia del género ni a los desiguales efectos económicos que puedan producirse, como sí ha evidenciado la literatura (Jacobs, 2001).

Por otro lado, se configura el "tabaquismo pasivo" como un aspecto fundamental del consumo de tabaco como problema de salud pública. Aquí sí que aparecen las mujeres, como "fumadoras pasivas" o "involuntarias" -junto a otros grupos vulnerables como personas ancianas, enfermas, niños y niñas-, pero, sobre todo, como causantes de ese "tabaquismo pasivo" en su doble condición de embarazadas y fumadoras. El discurso hegemónico de los planes analizados apunta hacia la definición del "tabaquismo materno" como el principal problema que afecta específicamente a las mujeres. Se tiende a hacer equivalente la salud de las mujeres con su salud reproductiva, permaneciendo ocultos o infraestimados otros problemas para la salud de las mujeres fumadoras que no están directamente relacionados con la maternidad. Además, se mide este impacto no tanto sobre la propia salud de la fumadora embarazada sino sobre la salud del feto:

El tabaquismo materno durante el embarazo tiene efectos negativos para el desarrollo del feto y el estado de salud del neonato. Se presentan elevados riesgos de bajo peso al nacer, prematuridad (ambos directamente relacionados con la cantidad diaria de cigarrillos fumados) aborto, ingreso neonatal en U.C.I., muerte perinatal y síndrome de muerte súbita (AND-05/10).

Una consecuencia de esto es la solidificación de un doble estándar, pues en los planes de tabaquismo la variable "tabaquismo materno" no tiene su equivalente masculino, aunque algunos de estos textos, como se describirá más adelante, aluden a las parejas de las mujeres embarazadas.

\subsection{Diagnóstico: Cuando la desagregación por sexo no es suficiente}

En los planes estudiados, se observa un esfuerzo por incorporar la realidad de mujeres y hombres en sus análisis de la situación, aunque la desagregación por sexo de los datos es todavía parcial y no suele profundizar en la interacción del sexo 
con otras variables relevantes al consumo de tabaco, como la clase social, el origen geográfico o la nacionalidad. Predomina un enfoque eminentemente epidemiológico centrado exclusivamente en datos sobre prevalencia y morbimortalidad, obviando en general otras variables importantes de motivación y de contexto que podrían ayudar a entender mejor las experiencias y necesidades de mujeres y hombres en relación al tabaco así como sus condicionamientos sociales y de género. En el cuadro 3 , se señalan los planes que incluyen indicadores de diagnóstico desagregados y específicos por sexo, así como su interrelación con otras variables.

Son escasas las referencias a factores sociales que pueden afectar de forma diferencial a mujeres y hombres y que podrían desvelar los sentidos que están detrás de los indicadores epidemiológicos de inicio, continuación y abandono del consumo de tabaco (Jiménez Rodrigo, 2011, 2012). No obstante, se incluyen algunas referencias a encuestas de opinión sobre el consumo de tabaco, pero cuyos resultados se muestran pocas veces desagregados por sexo. Paradójicamente, y en contraste a la hipervisibilización de las mujeres embarazadas tanto en la representación del problema del "tabaquismo materno" como en la configuración de los "grupos de riesgo", son excepcionales los planes que incorporan cifras acerca de las experiencias de las mujeres embarazadas con el tabaco (AND-05/10).

Cuadro 3. Desagregación por sexo y por otras categorías sociodemográficas de las variables de diagnóstico $(\mathrm{n}=11)$

Variables incluidas en el Planes diagnóstico

\begin{tabular}{|c|c|c|c|}
\hline \multirow[t]{5}{*}{$\begin{array}{l}\text { Uso del sexo } \\
\text { como } \\
\text { categoría } \\
\text { única }\end{array}$} & \multirow[t]{5}{*}{$\begin{array}{l}\text { Desagregación } \\
\text { por sexo }\end{array}$} & Morbilidad y mortalidad & $\begin{array}{l}\text { NAC-03/07, AND-02, } \\
\text { AND-05/10, BAL-03/07, } \\
\text { CAN04-07, CAN-08/11, } \\
\text { CAN-12/16, MAD- } \\
\text { 05/07, RIO-03/09 }\end{array}$ \\
\hline & & $\begin{array}{l}\text { Prevalencia } \\
\text { características } \\
\text { consumo }\end{array}$ & $\begin{array}{lr}\text { NAC-03/07, } & \text { AND-02, } \\
\text { AND-05/10, AST-09/12, } & \text { AST-03/07, CAN03-07, } \\
\text { BAL-0 CAN- } \\
\text { CAN-08/11, } \\
\text { 12/16, CEU-13-17, } \\
\text { CLM-02/07, MAD- } \\
\text { 05/07, RIO-03/09 }\end{array}$ \\
\hline & & $\begin{array}{l}\text { Prevalencia de abandono } \\
\text { del consumo }\end{array}$ & $\begin{array}{c}\text { CAN-08/11, } \text { CAN-12/16, } \\
\text { MAD-05/07, RIO-03/09 }\end{array}$ \\
\hline & & $\begin{array}{l}\text { Motivos de consumo e } \\
\text { intención } \\
\text { abandono }\end{array}$ & AND-05/10, CAN-08/11 \\
\hline & & $\begin{array}{c}\text { Actitudes frente al } \\
\text { consumo de tabaco }\end{array}$ & CAN-08/11 \\
\hline
\end{tabular}




\begin{tabular}{|c|c|c|c|}
\hline & & $\begin{array}{c}\text { Exposición al humo } \\
\text { ambiental de tabaco }\end{array}$ & AST-09/12, CAN-12/16 \\
\hline & Sólo mujeres & $\begin{array}{l}\text { Prevalencia mujeres } \\
\text { embarazadas }\end{array}$ & AND-05/10 \\
\hline & & Prevalencia de consumo & $\begin{array}{lr}\text { NAC-03/07, } & \text { AND-05/10, } \\
\text { BAL-03/07, } & \text { CAN04-07, } \\
\text { CAN-12/16, CLM- } & \text { CL } \\
\text { 02/07, } & \text { MAD-05/07, } \\
\text { RIO-03/09 } & \end{array}$ \\
\hline & & Mortalidad & AND-02 \\
\hline & Edad & $\begin{array}{l}\text { Abandono de consumo } \\
\text { de tabaco }\end{array}$ & MAD-05/07 \\
\hline $\begin{array}{l}\text { categorías } \\
\text { múltiples } \\
\text { en } \\
\text { interrelaci } \\
\text { ón con el } \\
\text { sexo }\end{array}$ & & $\begin{array}{l}\text { Inicio/prevalencia } \\
\text { consumo } \\
\text { jóvenes/adolescentes }\end{array}$ & $\begin{array}{l}\text { AND-02, AND-05/10, AST- } \\
\text { 09/12, BAL-03/07, } \\
\text { CAN04-07, CAN-08/11, } \\
\text { CAN-12/16, CEU-13-17, } \\
\text { CLM-02/07, MAD- } \\
\text { 05/07, RIO-03/09 }\end{array}$ \\
\hline & & $\begin{array}{c}\text { Actitudes frente } \\
\text { abandono jóvenes }\end{array}$ & $\begin{array}{c}\text { AND-05/10, } \\
\text { CEU-13-17 }\end{array}$ \\
\hline & & Situación laboral & AND-05/10 \\
\hline & Nivel & Renta & AND-05/10, MAD-05/07 \\
\hline & & Nivel de estudios & AND-05/10, MAD-05/07 \\
\hline & & Clase social & AND-05/10, MAD-05/07 \\
\hline
\end{tabular}

Fuente: Elaboración propia

Un problema de no ir más allá de las variables epidemiológicas desagregadas por sexo y de la descontextualización de los diagnósticos es la reproducción de espejismos de igualdad, llegándose a afirmar la "desaparición de las diferencias entre hombres y mujeres en el consumo de tabaco, pues [...] la prevalencia de consumo diario es la misma en hombres que en mujeres" (CAN-0407). Este planteamiento puede ser muy nocivo para el avance efectivo hacia la igualdad de género en salud, pues la convergencia estadística puede ocultar procesos sociales desiguales subyacentes.

Además de contemplar las diferencias entre los sexos en cuanto a prevalencias y pautas de inicio y abandono, es fundamental reinsertar estos indicadores en las coordenadas socioculturales y de género para una mayor comprensión de la situación y un mejor diseño de las acciones de intervención. Así, la problemática del consumo de tabaco en ámbitos como el educativo o el laboral se 
describe de forma ciega al género, sin profundizar en cómo las diferentes posiciones de género pueden afectar al consumo de tabaco. Igualmente, las referencias incluidas en los planes sobre determinados colectivos profesionales -sobre todo los definidos como modélicos (profesionales de la salud y docentes) y profesiones con mayor exposición a humo ambiental de tabaco- no son planteados de forma específica por sexo, pese a la feminización de muchos de estos sectores. Así, por ejemplo, cuando se alude a los factores de riesgo derivados de la exposición al humo de tabaco en el sector de la hostelería, no se contemplan las desigualdades de género, pese a contar con una alta presencia femenina. Al mismo tiempo, se observa cierto androcentrismo en la selección de las profesiones con mayor riesgo cancerígeno frente al tabaco, como por ejemplo, empleos del sector industrial. Además, de los riesgos de exposición al humo de tabaco en el espacio laboral, algunos planes incorporan datos sobre el espacio doméstico, pero, nuevamente, las cifras desagregadas por sexo son insuficientes: no se muestran a las mujeres, cuando es un problema que afecta fundamentalmente a éstas, además de a niños y niñas (Wipfli et al., 2008).

Sin embargo, y pese a las limitaciones en la desagregación por sexo de algunas variables, es necesario enfatizar que la incorporación del género en el examen de las diferencias y desigualdades en salud no puede reducirse a este ejercicio de desdoblamiento de las estadísticas habituales. Es imprescindible incorporar, junto al sexo como variable descriptiva, otras variables relevantes al género, como las ligadas a la división sexual del trabajo, identidades y roles de género (Ruiz Cantero et al., 2006).

Desde un prisma interseccional, los planes examinados se caracterizan por el empleo mayoritario de categorías únicas -sexo y edad, principalmente, y en menor medida, nivel socioeconómico y nacionalidad- sin ser puestas en relación. Se produce así una sobregeneralización y la asunción de homogeneidad intragénero y, por tanto, una invisibilización interseccional de grupos cuya posición múltiple puede marcar específicas vulnerabilidades frente al tabaco. No obstante, en algunos planes (veáse cuadro 3) el sexo se cruza con otras variables, frecuentemente, la edad y, en menos casos, con variables socioeconómicas. Pero no podemos hablar de un uso de categorías interseccionales en los términos que establece Hancock (2007), estaríamos más bien ante un ejercicio de añadir y mezclar [add and stir] las diferencias, práctica que la interseccionalidad precisamente pone en cuestión y trata de superar (Guzmán, 2014). Aunque la mayoría de los planes incorpora en el análisis de la situación datos sobre el consumo de tabaco de adolescentes y jóvenes desagregados por sexo, apenas se consideran otras situaciones resultado de la intersección del género y la edad, como las relativas a las mujeres mayores donde se registran las mayores subidas de prevalencia de consumo de tabaco. Por otro lado, las condiciones socioeconómicas 
en interacción con el género como determinantes del consumo de tabaco son escasamente contempladas, pese a las evidencias disponibles en la literatura (Graham et al., 2006; Layte y Whelan, 2009).

\subsection{Caracterización de los grupos objetivo: El embarazo como dispositivo de definición de un "grupo de riesgo".}

Es habitual en los planes analizados la identificación de los "grupos de riesgo" o poblaciones diana en función de categorías únicas, de modo que las mujeres, junto a jóvenes y adolescentes, colectivos "modélicos" y otros grupos considerados como "vulnerables" (personas con enfermedad mental, hospitalizadas, reclusas), conforman un grupo aparte y separado de "especial atención" o "alto riesgo". Las mujeres son presentadas como un grupo homogéneo, compacto e impermeable a otras condiciones sociales más allá de la de ser mujer. Esta esencialización de las mujeres como grupo de riesgo está íntimamente ligada a la reducción biologicista de su salud a su dimensión sexual-reproductiva. La construcción del grupo de riesgo "mujeres" viene justificada por diferentes argumentos. Primero, por la incorporación al "hábito tabáquico" de las mujeres frente a la declinación del consumo masculino. Segundo, y en relación al anterior, por el incremento del cáncer de pulmón entre las mujeres. Y, tercero, por la identificación de "riesgos adicionales", "específicos de su género", que se refieren fundamentalmente a sus aspectos sexual-reproductivos:

Además de los riesgos generales asociados al tabaquismo, las mujeres fumadoras se enfrentan a un cierto número de riesgos adicionales para la salud específicos de su sexo, ya que el consumo de tabaco puede influir en su equilibrio hormonal. En concreto, el tabaquismo puede contribuir a una reducción de la fertilidad y a incrementar el riesgo de menopausia precoz. Además, es un factor que contribuye a la pérdida de calcio $\mathrm{y}$, por tanto, a la osteoporosis en las mujeres en fase postmenopáusica. El consumo de tabaco, cuando se combina con el uso de anticonceptivos orales, puede también incrementar sensiblemente el riesgo de desarrollar enfermedades cardiovasculares. Se ha asociado el consumo de tabaco durante el embarazo con un mayor riesgo de aborto espontáneo, complicaciones durante el embarazo, muerte fetal, partos prematuros, bajo peso al nacer y muerte del neonato. También aumenta el riesgo de muerte súbita del niño y se asocia con una serie de consecuencias a largo plazo en la salud del niño (por ejemplo, capacidad pulmonar reducida y mayores tasas de infección) (CLM-02/07).

Continúa prevaleciendo la configuración del "tabaquismo materno" en relación a los efectos perniciosos sobre la salud del feto y de las criaturas; lo que 
resulta en la paradoja de que el cuerpo de las mujeres, a pesar de ser hipervisibilizado por su condición de embarazadas, queda oculto e infraestimado tras las necesidades de sus hijos e hijas. Algunos planes, como el andaluz, reconocen la complejidad de esta problemática en relación a las presiones sociales que pueden desembocar en la estigmatización y culpabilización de las madres fumadoras:

Las embarazadas continúan fumando porque perciben beneficios inmediatos y anticipan una gran cantidad de costes ante la posibilidad de dejarlo. Además, están sometidas a múltiples demandas, que pueden llevar a vivir la situación como estresante y buscar alivio en el tabaco. Una cierta presión social existente ("fumar es una conducta perjudicial para el bebé") no se suele ver acompaña del apoyo necesario para facilitar el abandono. Pueden aparecer sentimientos de culpa en la mujer gestante que, en ocasiones, causan la infradeclaración de consumo, el ocultamiento del hábito o la tendencia a minimizar los riesgos potenciales para el bebé. La implantación, desde el sistema público de salud, de estrategias específicas para el abandono del tabaco en embarazadas puede incrementar significativamente los resultados en la reducción o eliminación del hábito en este grupo, que tiene, además, la peculiaridad de precisar dejar de fumar en un período muy corto de tiempo (AND-05/10).

Por otro lado, también se hace referencia a los efectos de la exposición al humo ambiental de tabaco durante la gestación -no proveniente de la propia embarazada sino del entorno-, si bien estos efectos son nuevamente focalizados en la criatura, no en la madre. Cabe destacar que en algunos planes (AND-05/10, BAL-03/07, CAN-04-07, CAN-08/11) se hace mención al papel de la pareja: en algunos, como parte del problema; en otros, como parte de la solución.

Por otra parte solo el $30 \%$ de las mujeres fumadoras deja de fumar durante el periodo de gestación o de lactancia [...]. Además, se debería facilitar información, no solo de los perjuicios de consumir, también de los peligros de respirar ACHT, por lo que debería incluirse a las parejas fumadoras en los programas de atención a la embarazada (CAN-08/11).

No obstante, la responsabilidad de los padres aparece como secundaria, reforzando el estereotipo de considerar el cuidado de los hijos e hijas como una responsabilidad única o primariamente femenina:

El hecho de que la madre fume durante el embarazo aumenta un $50 \%$ las probabilidades de que el niño sufra este síndrome [de Muerte Súbita del lactante]. Este riesgo se incrementa si después del parto la madre sigue fumando. En este último caso influye también que el padre sea fumador, pero es mayor la influencia 
de la madre, probablemente porque los niños pequeños pasan más tiempo con sus madres (CAN-04-07).

Pese a los esfuerzos de algunos planes por desculpabilizar el proceso de abandono entre las madres fumadoras e involucrar a sus parejas, la definición del tabaquismo femenino en el embarazo está ligada a su patologización, estereotipificacion y estigmatización. El grupo de "mujeres embarazadas" aparece como grupo de riesgo junto a otros colectivos marcados por su desviación respecto a la norma social y sanitaria, como, por ejemplo, personas privadas de libertad, en situación de pobreza, o con trastornos mentales (AND-05/10).

Otros "riesgos específicos de su sexo" afectan también a su dimensión sexual reproductiva como los problemas en la menstruación, cánceres como el de cuello uterino, el uso de anticonceptivos orales y problemas de osteoporosis. Curiosamente, se resalta como "riesgo específico" la mayor preocupación estética de las mujeres por la halitosis, las manchas en los dedos y en los dientes, la aparición de más arrugas o el aspecto más apagado de la piel (CAN-04/07), contribuyendo así a la reproducción de estereotipos de género en relación al aspecto físico de las mujeres. En este mismo plan también se destaca el riesgo disfunción eréctil entre los varones, pero en ningún caso esta circunstancia conforma grupos de riesgo ni la patologización de su práctica tabáquica.

\subsection{Objetivos y medidas de intervención: El silencio de los planes sobre otras necesidades de las mujeres fumadoras más allá del embarazo}

Como muestran otros estudios que han analizado la incorporación de la perspectiva de género en las políticas públicas, es más común encontrar en los planes y otros documentos de implementación la referencia a las diferencias de género en sus diagnósticos que en otros componentes de sus diseños (Haar y Verloo, 2013: 422). En el caso que nos ocupa, esta preocupación por las diferencias en función del sexo no se traduce de forma proporcional en la formulación de objetivos y medidas específicas y/o sensibles al género (cuadro 4). Algunos planes reconocen la necesidad de atender al género "en todas las actuaciones" (CAN08/11), "en los programas de atención y deshabituación" (CAN-12/16) y "en el diseño e implementación de las intervenciones preventivas y asistenciales" (CEU13/17). En tal sentido, se afirma la relevancia del género y las circunstancias específicas del consumo de tabaco entre las mujeres:

El tabaquismo incrementa la desigualdad de género en salud. Presenta caracteristicas específicas que han de tomarse en cuenta en la prevención y en la asistencia para dejar de fumar. Las mujeres pueden requerir un abordaje 
especifico, que tenga en cuenta de forma diferenciada sus necesidades $y$ expectativas (CAN-12/16).

Muchos más planes incorporan objetivos específicos al sexo cuando buscan la prevención e intervención con mujeres embarazas (AND-05/10, CAN-08/11, CLM-02/07, RIO-09/13), la reducción de la prevalencia de consumo en chicas jóvenes (CAN-08/11) o la deshabituación entre las mujeres (CAN-08/11). Otros espacios de actuación recogidos en los planes analizados como los de participación o el normativo no incorporan objetivos significativos al género. Los varones no se recogen en el cuadro 4, pues no son identificados como población de "especial atención" en ninguna de las medidas recogidas en los planes examinados. Tampoco se consideran las necesidades particulares que pudieran tener diferentes grupos de varones y mujeres en función de la clase social o el origen nacional o étnico.

Cuadro 4. Sensibilidad y especificidad al género de las medidas y actuaciones de los planes analizados sobre control de tabaquismo

\begin{tabular}{|c|c|c|c|c|}
\hline \multirow[t]{2}{*}{ Ámbito } & \multirow{2}{*}{$\begin{array}{l}\text { Medidas } \\
\quad \text { sensibles al } \\
\quad \text { género }\left(n^{o}\right)\end{array}$} & \multicolumn{3}{|c|}{ Medidas específicas a las mujeres $\left(n^{\circ}\right)$} \\
\hline & & En general & Mujeres jóvenes & $\begin{array}{l}\text { Mujeres } \\
\quad \text { embarazad } \\
\text { as }\end{array}$ \\
\hline Prevención & NAC-03/07 (1) & -- & -- & -- \\
\hline $\begin{array}{l}\text { Formación } \\
\quad \text { profesionales }\end{array}$ & -- & NAC-03/07 (2) & NAC-03/07 (1) & -- \\
\hline $\begin{array}{l}\text { Sensibilización/ } \\
\text { Comunicación }\end{array}$ & RIO-09/13 (1) & $\begin{array}{l}\text { NAC-03/07 (1) } \\
\text { AST-09/12 (1) } \\
\text { CAN-04/07 (1) } \\
\text { CAN-08/11 (2) } \\
\text { RIO-09/13 (2) }\end{array}$ & $\begin{array}{l}\text { AND-02 (1) } \\
\text { AST-09/12 (1) }\end{array}$ & $\begin{array}{l}\text { CAN-04/07 (2) } \\
\text { CEU-13/17 (1) } \\
\text { RIO-09/13 (3) }\end{array}$ \\
\hline Intervención & $\begin{array}{l}\text { CEU-13/17 (1) } \\
\text { AND-05/10 (1) }\end{array}$ & $\begin{array}{l}\text { CAN-04/07 (1) } \\
\text { CEU-13/17 (1) }\end{array}$ & -- & $\begin{array}{l}\text { NAC-03/07 (1) } \\
\text { CAN-04/07 (1) } \\
\text { MAD-05/07 } \\
\quad(1) \\
\text { RIO-03/09 (2) }\end{array}$ \\
\hline Investigación & $\begin{array}{l}\text { NAC-03/07 (4) } \\
\text { CAN-04/07 } \\
\text { (1) }\end{array}$ & $\begin{array}{l}\text { AND-05/10 (1) } \\
\text { CAN-04/07 (1) }\end{array}$ & -- & $\begin{array}{l}\text { AND-05/10 (1) } \\
\text { CAN-04/07(1) } \\
\text { RIO-03/09 (1) }\end{array}$ \\
\hline
\end{tabular}

Fuente: Elaboración propia

El ámbito de la sensibilización es el que más medidas incorpora en relación a las diferencias de género y a las necesidades específicas de las mujeres. De nuevo, 
son dos los "colectivos" que concentran los mayores esfuerzos de sensibilización: mujeres jóvenes y mujeres embarazadas.

Las medidas de intervención son las que más se centran en el "tabaquismo materno", estableciendo protocolos específicos en atención primaria, obstetricia y ginecología así como de derivación a unidades especializadas de tabaquismo. Se observa como en el caso de las mujeres embarazadas se produce un proceso de doble de patologización y medicalización: uno derivado de su condición de embarazadas; y otro, de su práctica de fumar. En este sentido, también se trata de potenciar en los planes la investigación sobre las mujeres, especialmente en situaciones de gestación y postparto, constatadas las dificultades de las mujeres embarazadas para dejar de fumar. Sin embargo, nada se apunta en relación a los varones. Y tampoco se contemplan posibles condiciones sociales que puedan relacionarse con el género y marcar experiencias diversas en relación al embarazo y el consumo de tabaco.

\subsection{Evaluación: La falta de indicadores sensibles al género}

El examen de los procedimientos de evaluación previstos en los planes examinados revela el predominio de enfoques cuantitativos así como la presencia limitada de indicadores desagregados o específicos por sexo (cuadro 5). Los indicadores planteados en relación al sexo se orientan fundamentalmente a la medición de las acciones desarrolladas en su implementación y a la medición de la prevalencia de consumo de tabaco. Esta escasez de indicadores de evaluación sensibles al género puede derivar en un entendimiento parcial e incompleto de los efectos de las políticas sobre tabaco en mujeres y en hombres, imposibilitando la generación de elementos que puedan contribuir a su mejora y a la atención de las necesidades específicas de las mujeres, más allá de su condición joven o gestante; pero también la de los hombres.

Cuadro 5. Indicadores desagregados y específicos por sexo incluidos en los planes analizados sobre control de tabaquismo

\begin{tabular}{|c|c|c|}
\hline $\begin{array}{c}\text { Ámbito de } \\
\text { evaluación }\end{array}$ & $\begin{array}{l}\text { Indicadores desagregados por } \\
\text { sexo }\end{array}$ & especificos por \\
\hline Implementación & 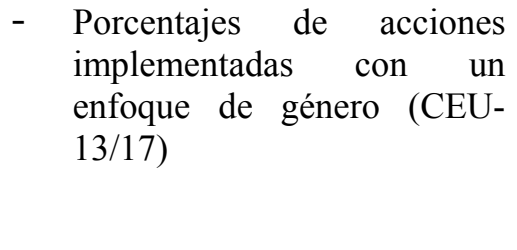 & $\begin{array}{ll}\text { - } & \begin{array}{l}\text { Actuaciones realizadas } \\
\text { específicamente a mujeres }\end{array} \\
\text { (CAN-08/11, RIO-09/13) } \\
\text { - } & \begin{array}{l}\text { Actuaciones realizadas } \\
\text { específicamente a mujeres } \\
\text { embarazadas (CAN-08/11, }\end{array}\end{array}$ \\
\hline
\end{tabular}




\begin{tabular}{|c|c|c|}
\hline & & CAN-12/16, RIO-09/13) \\
\hline Efectos & 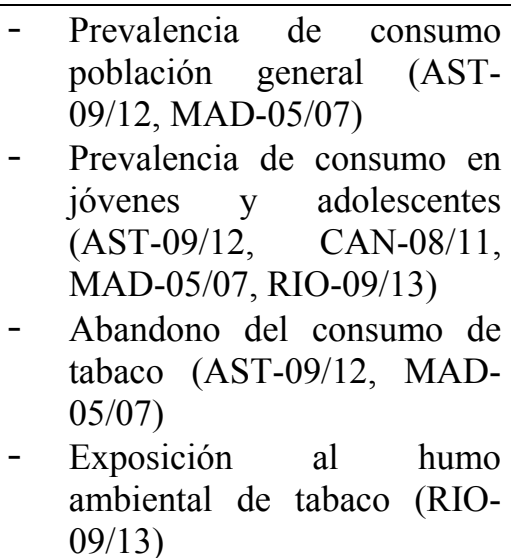 & 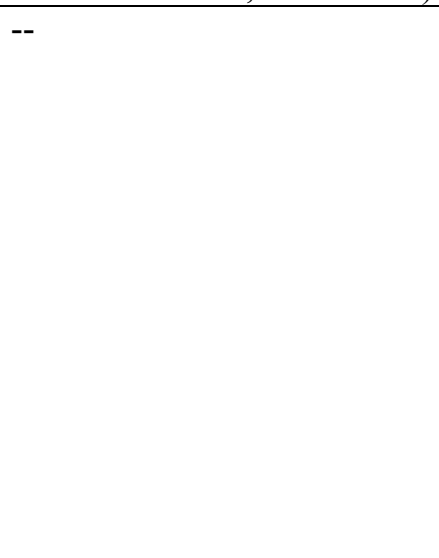 \\
\hline
\end{tabular}

Fuente: Elaboración propia

\section{CONCLUSIONES}

El análisis de los planes para la prevención e intervención sobre el tabaquismo sirve de (pre)texto para reflexionar sobre los sesgos de género que afectan a las políticas públicas en salud y que pueden desembocar en efectos desiguales en mujeres y hombres. El diseño de los planes sobre el tabaquismo, pese a la creciente incorporación de datos desagregados por sexo en el diagnóstico, se ven adolecidos por sesgos de género que pueden limitar tanto la eficacia como la equidad de sus actuaciones. Se observa un creciente reconocimiento de las diferencias entre mujeres y hombres en el análisis de la situación -sobre todo, en cuanto a la descripción de las prevalencias de consumo y tasas de morbimortalidad$\mathrm{y}$ una preocupación hacia el incremento del número de fumadoras. Sin embargo, no se refleja de forma proporcional en medidas específicas y adaptadas a las necesidades de la población femenina más allá del embarazo, ni tampoco en la selección de los indicadores para la medición de la eficacia y la equidad de las intervenciones. La desagregación por sexo, aunque fundamental para avanzar en el entendimiento de las desigualdades de género, no es suficiente. Es imprescindible incorporar indicadores sensibles al género que permitan contextualizar las diferencias y la desigualdades en salud entre mujeres y hombres, pero también escapar de representaciones binarias (Nowatzki y Grant, 2011).

El tratamiento de las experiencias de las mujeres en relación al tabaco se ve afectado por la influencia de dos tipos de representaciones que -aunque aparentemente se mueven en direcciones contrarias- convergen y se refuerzan mutuamente ofreciendo una imagen de la salud de las mujeres parcial y 
estereotipada. Por una parte, se produce una invisibilización de la situación de las mujeres, como consecuencia de un androcentrismo implícito, sostenido por el uso de indicadores globales y el uso del masculino como genérico en algunos planes, que termina vinculando el consumo de tabaco con enfermedades fuertemente masculinizadas, como son el cáncer de pulmón y las dolencias cardiovasculares. Por otra parte, se da una hipervisualización de las mujeres en una esfera muy concreta: la sexual-reproductiva, donde los varones no están presentes, salvo en unos pocos planes que aluden a la importancia de la pareja. Esta visión se proyecta en la transmisión de dobles estándares y estereotipos de género acerca del rol de madres de las mujeres fumadoras, en virtud de los cuales, se termina conformando el problema del "tabaquismo femenino" en torno al embarazo y sus efectos sobre el feto. Como afirmó Simone de Beauvoir (1949/2000), los roles y proyectos de las mujeres se entenderían así como proyectos proyectados, recurrentemente vinculados a la inesencialidad-alteridad de ser madre-de, ser mujer-de.

Esta reducción de la salud de las mujeres tiene consecuencias prácticas y en la organización de las relaciones sociales: por ejemplo, en la atención sanitaria, que puede limitar sus intervenciones dirigidas a las mujeres a cuando éstas queden embarazadas, ignorando las necesidades de las mujeres fumadoras más allá de la maternidad. No es extraño que en la práctica que una porción sustancial de mujeres fumadoras sólo se plantee o decida dejar el tabaco durante del embarazo, percibiéndolo como un asunto estacional -circunscrito a los meses de gestación y, posiblemente, al período de lactancia-y reversible (Jiménez Rodrigo, 2011, 2012). El fracaso de muchas mujeres en su intento por dejar el consumo de tabaco antes, durante y después del embarazo pone de relieve el desconocimiento de los determinantes del consumo de cigarrillos entre las mujeres embarazadas y la ineficacia de las estrategias institucionales sanitarias (Khan, Certain y Whitetaker, 2002). Por ello, es básico seguir avanzando en la investigación de las experiencias y las necesidades de las mujeres fumadoras durante su embarazo que pueden obstaculizar la puesta en práctica de su decisión de dejar fumar, pero también es preciso romper la relación entre el consumo de tabaco en las mujeres respecto a su dimensión reproductiva para reintegrarla en un modelo más social y holístico, huyendo de su patologización y estigmatización.

Dado que un objetivo central de las políticas públicas en el contexto de las sociedades democráticas avanzadas es cubrir las necesidades de los grupos socioeconómicamente desfavorecidos y vulnerables, es urgente detectar y atender a aquellos perfiles con mayor riesgo de empezar a fumar y de no poder dejarlo. Es el caso de chicas adolescentes y jóvenes, mujeres mayores de 45 años, mujeres con pocos recursos, de origen inmigrante o pertenecientes a minorías étnicas; pero también el de determinados perfiles de varones que, pese al decrecimiento general 
en sus tasas de consumo de tabaco, presentan altas cifras de prevalencia, como los de clases trabajadoras o en situación de desempleo.

Una mirada de género interseccional contribuiría a la identificación, entendimiento e inclusión en las políticas públicas de las diversas y desiguales situaciones en relación a la salud que pueden darse como resultado de la intersección de múltiples ejes de privilegio y marginación en función de la edad, clase social, raza/etnia, origen geográfico, nacionalidad, (dis)capacidad, entre otros. Esta propuesta no debe entenderse simple y únicamente como una demanda feminista, sino como una necesidad fundamental de equidad, pues contribuye a asegurar beneficios equivalentes para el conjunto de la población y la integración de colectivos marginalizados en su campo de actuación. Pero también de eficacia y eficiencia, al mejorar el logro de los objetivos buscados por las políticas en salud así como la ampliación de la cobertura de sus efectos. La articulación de equidad y eficacia son cuestiones ahora más fundamentales que nunca en un contexto de creciente desigualdad social y de restricciones en la inversión pública en salud. Por ello, los marcos de género y el enfoque interseccional emergen como decisivas y potentes herramientas para enfrentar los problemas, necesidades y retos que imponen los tiempos de crisis.

\section{BIBLIOGRAFÍA}

AMOS, Amanda ; GREAVES, Lorraine, NICHTER, Mimi, \& BLOCH, Michel (2012): Women and tobacco: a call for including gender in tobacco control research, policy and practice. Tobacco Control, 21, 236-243. doi:10.1136/tobaccocontrol-2011-050280

ASTELARRA, Judith (2004): Politicas de género en la Unión Europea y algunos apuntes sobre América Latina. CEPAL, Unidad Mujer y Desarrollo. http://mediterraneas.org/IMG/pdf/lcl2154e.pdf Acceso: 15 de diciembre de 2014.

BAUER, Greta (2014): Incorporating intersectionality theory into population health research methodology: challenges and the potential to advance health equity. Social Science and Medicine, 11, pp. 10-7. doi: 10.1016/j.socscimed.2014.03.022

BARKER, Kristin (1998): A ship upon a stormy sea: The medicalization of pregnancy. Social Science and Medicine, 47.8, pp. 1067-76.

BEAUVOIR, Simone (1949/2000): El segundo sexo. Los hechos y los mitos. Madrid: Cátedra.

BERRIDGE, Virginia (2001): Constructing Women and Smoking as a Public Health Problem in Britain: 1950-1990s. Gender \& History, 13(2), pp. 328-48. 
BIRD, Chloe \& RIEKER Patricia (1999): Gender matters: an integrated model for understanding men's and women's health. Social Science and Medicine, 48, pp. 745-755. doi: 10.1016/S0277-9536(98)00402-X

BRËDSTROM, Anna (2006): Intersectionality: A Challenge for Feminist HIV/AIDS Research? European Journal of Women's Studies, 13(3), pp. 229-243. doi: $10.1177 / 1350506806065754$

BOWLEG, Lisa (2012): The problem with the phrase women and minorities: intersectionality - an important theoretical framework for public health. American Journal of Public Health, 102(7), pp. 1267-1273. doi: 10.2105/AJPH.2012.300750

BUSTELO, María \& LOMBARDO, Emanuela (2007): ¿Qué hay debajo de la alfombra de las políticas de igualdad? Un análisis de 'marcos interpretativos' en España y en Europa. En BUSTELO, María \& LOMBARDO, Emanuela (eds.) Políticas de igualdad en España y en Europa. Afinando la mirada, pp. 11-36. Madrid: Cátedra.

CAHILL, Heather (2001): Male appropriation and medicalization of childbirth: an historical analysis. Journal of Advanced Nursing, 33(3), pp. 334-42.

CHRISTOFIDES, Nicola (2002): How to Make Policies More Gender Sensitive. En: SAMET, Jonathan \& YOON, Soon-Young (eds.) Women and the Tobacco Epidemic. Challenges for Epidemic-Challenges for the 21st Century, pp. 16576. Geneva: World Health Organization.

CRENSHAW, Kimberle (1991): Mapping the margins: Intersectionality, identity politics, and violence against women of color. Stanford Law Review, 43(6), pp. 1241-1299.

DEDOBBELEER, Nicole; BÉLAND, François; CONTANDRIOPOULOS, AndréPierre \& ADRIAN, Manuella (2004): Gender and the Social Context of Smoking Behaviour. Social Science \& Medicine, 58(1), pp. 1-12.

DOLL, Richard; PETO, Richard; WHEATLEY, Keith; GRAY, Richard \& SUTHERLAND, Isabelle (1994): Mortality in relation to smoking: 40 years' observations on male British doctors. British Medical Journal, 309, pp. 901-11.

DOYAL, Lesley (2000): Gender equity in health: debates and dilemmas. Social Science and Medicine, 51, pp. 931-939. doi: 10.1016/S0277-9536(00)00072-1

DOYAL, Lesley (2009): Challenges in researching life with HIV/AIDS: an intersectional analysis of black African migrants in London. Culture, Health \& Sexuality, 11(2), pp. 173-188. doi: 10.1080/13691050802560336

EICHLER, Margrit (1999): Nonsexist Research Methods. A Practical Guide. New York: Routledge.

EICHLER, Margrit \& BURKE, Mary Anne (2006): The BIAS FREE Framework. A new analytical tool for global health research. Canadian Journal of Public Health, 97(1), pp. 63-68.

GARCÍA CALVENTE, María del Mar, JIMÉNEZ RODRIGO, María Luisa; Martínez MORANTE, Emilia (2010): Guía para incorporar la perspectiva de género a la investigación en salud. Granada: Escuela Andaluza de Salud Pública. 
GARCÍA PRINCE, Evangelina (2008): Politicas de igualdad, equidad y gender mainstreaming. ¿De qué estamos hablando? Marco conceptual. Fondo EspañaPNUD.

http://www.americalatinagenera.org/es/documentos/doc 732_Politicasdeiguald ad23junio08.pdf.

GRAHAM, Hilary; FRANCIS, Brian; INSKIP, Hazel \& HARMAN, Juliet (2006): Socioeconomic lifecourse influences on women's smoking status in early adulthood. Journal of Epidemiology and Community Health, 60, pp. 228-233. doi:10.1136/jech.2005.039784

GREAVES, Lorraine \& BARR, Victoria (2000): Filtered policy: Women and tobacco in Canada. Vancouver, BC: British Columbia Centre of Excellence for Women's Health.

GREAVES, Lorranie \& JATEGAONKAR, Natasha (2006): Tobacco policies and vulnerable girls and women: Toward a framework for gender sensitive policy development. Journal of Epidemiology and Community Health, 60(Suppl. II), pp. 57-65. doi: 10.1136/jech.2005.045393

GUZMÁN ORDAZ, Raquel (2014): El paradigma interseccional: rutas teóricometodológicas para el análisis de las desigualdades sociales. En SALETTI, Lorena (Coord.): Traslaciones en los estudios feministas. Málaga: Editorial Perséfone - Universidad de Málaga.

HAAR, Marleen \& VERLOO, Mieke (2013): Unpacking the Russian doll: gendered and intersectionalized categories in European gender equality policies. Politics, Groups, and Identities, 1(3), pp. 417-432. http://dx.doi.org/10.1080/21565503.2013.816246

HANCOCK, Ange-Mary (2007): When multiplication doesn't equal quick addition: Examining intersectionality as a research paradigm. Perspectives on Politics, 5(1), pp. 63-79. http://dx.doi.org/10.1017/S1537592707070065

HANKIVSKY, Olena (2012): Women's health, men's health, and gender and health: implications of intersectionality. Social Science \& Medicine, 74(11), pp. 17121720. doi: 10.1016/j.socscimed.2011.11.029

HIRSCHFELDER, Arlene (1999): Encyclopaedia of Smoking and Tobacco. Phoenix: Oryx Press.

IM, Eun-Ok (2000): A feminist critique of research on women's work and health. Health Care for Women International, 21, pp. 105-19. doi: $10.1080 / 073993300245339$

INHORN, Marcia \& WHITTLE, Lisa (2001): Feminism meets the "new" epidemiologies: Toward an appraisal of antifeminist biases in epidemiological research on women's health. Social Science \& Medicine, 53, pp. 553-67. doi: 10.1016/S0277-9536(00)00360-9

JACOBS, Rowena (2001): Economic Policies, Taxation and Fiscal Measures. En: SAMET, Jonathan \& YOON, Soon-Young (eds.) Women and the Tobacco Epidemic. Challenges for Epidemic-Challenges for the 21st Century, pp. 177200. Geneva: World Health Organization. 
JAGSI, Reshma; MOTOMURA, Amy; AMARNATH, Sudah; JANKOVIC, Aleksandra; SHEETS, Nathan \& UBEL, Peter (2009): Under representation of women in high impact published clinical cancer research. Cancer, 115(14), pp. 3293-3301. doi: $10.1002 /$ cncr.24366

JIMENEZ RODRIGO, María Luisa (2010): La feminización del consumo de tabaco: ¿Convergencia o desigualdad? Revista española de drogodependencias, 35(3), pp. 285-296.

JIMENEZ RODRIGO, María Luisa (2011): Mujeres, abandono de tabaco y barreras de género. Feminismo/s, 18, pp. 39-66.

JIMENEZ RODRIGO, María Luisa (2012): De antorcha de libertad a estigma: resignificaciones del consumo femenino de tabaco. Clepsydra. Revista de Estudios de Género y Teoría Feminista, 11, pp. 79-101.

JIMENEZ RODRIGO, María Luisa y GUZMÁN ORDAZ, Raquel (2012): Género y usos de drogas: dimensiones de análisis e intersección con otros ejes de desigualdad. Oñati Socio-Legal Series, 2(6), pp. 77-96.

KHAN, Robert; CERTAIN, Laura \& WHITETAKER, Robert (2002): A reexamination of smoking before, during, and after pregnancy. American Journal of Public Health, 92(11), pp. 1801-8.

LAPARRA, Miguel (coord.) (2011): Diagnóstico social de la comunidad gitana en España. Un análisis contrastado de la Encuesta del CIS a Hogares de Población Gitana 2007. Madrid: Ministerio de Sanidad, Política Social e Igualdad.

LAYTE, Richard \& WHELAN, Christopher (2009): Explaining social class inequalities in smoking: The role of education, self-efficacy, and deprivation. European Sociology Review, 25, pp. 399-410. doi: 10.1093/esr/jen022

LOMBARDO, Emanuela; MEIER, Petra \& Verloo, Mieke (2012): Policymaking and Gender. FREIA Working Paper Series, $\mathrm{N}^{\circ} .81$. http://eurogender.eige.europa.eu/sites/default/files/freia wp 81.pdf. Acceso: 21 de diciembre de 2014.

LOMBARDO, Emanuela \& ROLANDSEN AGUSTÍN, Lise (2012): Framing gender intersections in the European Union: What implications for the quality of intersectionality in policies? Social Politics: International Studies in Gender, State \& Society, 19(4), pp. 482-512. doi: 10.1093/sp/jxr001

NIEUWENHOVEN, Linda \& KLINGE, Ineke (2010): Scientific excellence in applying sex-and gender-sensitive methods in biomedical and health research. Journal of Women's Health, 19(2), pp. 313-321. doi: 10.1089/jwh.2008.1156

NOWATZKI, Nadine; GRANT, Karen (2011): Sex is not enough: The need for genderbased analysis in health research. Health care for women international, 32(4), pp. 263-277. doi: 10.1080/07399332.2010.519838

OAKLEY, Ann (1989): Smoking in pregnancy: smokescreen or risk factor? Toward a materialist analysis. Sociology of Health and Illness, 11(4), pp. 311-34. doi: 10.1111/1467-9566.ep11372513

ORTIZ, Teresa (2006): Medicina, historia y género. 130 años de investigación feminista. Oviedo: KRK. 
PEIRÓ, Rosana; RAMÓN, Nieves; ÁlVAREZ-DARDET, Carlos; COLOMER, Concha; MOYA, Carmela; BORRELL, Carme et al. (2004): Sensibilidad de género en la formulación de planes de salud en España: lo que pudo ser y no fue. Gaceta Sanitaria, 18(Supl 2), pp. 36-46.

PLAN NACIONAL SOBRE DROGAS (PNSD) (2010): Encuesta estatal sobre uso de drogas en enseñanzas secundarias (ESTUDES) 1994-2010. http://www.pnsd.msssi.gob.es/Categoria2/observa/estudios/home.htm Acceso: 10 de junio de 2014.

ROGGEBAND Conny \& VERLO Mieke (2007): A closer look at the intersection of gender equality and migration in the Netherlands (1995-2005). En Verlo, Mieke (ed.): Multiple meanings of gender equality. A critical frame analysis of gender policies in Europe, pp. 257-280. Budapest: Central European University Press.

RUIZ CANTERO, María Teresa \& VERDÚ-DELGADO, María (2004): Sesgo de género en el esfuerzo terapéutico. Gaceta Sanitaria, 18(1), pp. 118-125.

RUIZ CANTERO, M. Teresa \& VERBRUGGE, Lois M. (1997): A two way view of gender bias in medicine. Journal of Epidemiology and Community Health, 51(2), pp. 106-109. doi:10.1136/jech.51.2.106

RUIZ CANTERO, M. Teresa; PAPÍ, Natalia; CABRERA, Virginia; RUIZ MARTÍNEZ, Ana \& ÁLVAREZ-DARDET, Carlos (2006): Los sistemas de género y/en la Encuesta Nacional de Salud. Gaceta Sanitaria, 20(6), pp. 427434.

SÁNCHEZ DE COS, Julio (2009): El cáncer de pulmón en España. Epidemiología, supervivencia y tratamientos actuales. Archivos de Bronconeumonología, 45(7), pp. 341-48. doi: 10.1016/j.arbres.2008.06.005

SCHNEIDER, Anne \& INGRAM, Helen (1993): Social construction of target populations: implications for politics and policy. The American Political Science Review, 87(2), pp. 334-347.

SEN, Gita; ÖSTLIN, Piroska; GEORGE, Asha (2005): Incorporar la perspectiva de género en la equidad en salud: un análisis de la investigación y las políticas. Pan American Health Org. www.catunescomujer.org/catunesco_mujer/.../PO 14 entire book.pdf Acceso: 10 de junio de 2014.

STONE, Deborah 1989. Causal stories and the formation of policy agendas. Political Science Quarterly, 104 (2), pp. 281-300.

SUBIRATS, Joan; KNOEPFEL, Peter; LARRUE, Corinne \& VARONE, Frédéric (2008): Análisis y gestión de políticas públicas. Barcelona: Ariel.

VALLS, Carme (2009): Mujeres, salud y poder. Madrid: Catedra.

WALDRON, Ingrid (1991): Patterns and causes of gender differences in smoking. Social Science \& Medicine, 32(9), pp. 989-1005.

WELDON, Laurel (2006): The structure of intersectionality: A comparative politics of gender. Politics \& Gender, 2(2), pp. 235-248. http://dx.doi.org/10.1017/S1743923X06231040 
WIPFLI, Heather; AVILA-TANG, Erika; NAVAS-ACIEN, Ana; KIM, Sungroul; ONICESCU, Georgiana; YUAN, Jie et al. (2008): Secondhand smoke exposure among women and children: Evidence from 31 countries, American Journal of Public Health, 98(4), pp. 672-79. doi: 10.2105/AJPH.2007.126631

WORLD HEALTH ORGANIZATION (1999): Making a difference to tobacco and health: Avoiding the tobacco epidemic in women and youth. WHO International Conference on Tobacco and Health. Kobe Japan, 14 - 18 November 1999. Kobe: WHO.

WORLD HEALTH ORGANIZATION (2007): Gender and tobacco control: A policy brief. Switzerland: WHO. 